\title{
ASSESSMENT OF LEFT VENTRICULAR FUNCTION USING 12-LEAD ECG AND CARDIAC TROPONIN-T IN CORRELATION WITH 2D-ECHO FOLLOWING NEW-ONSET MYOCARDIAL INFARCTION
}

\author{
Peddi Bhaskar1, Bikshapathi Rao², Naveen ${ }^{3}$
}

${ }^{1}$ Associate Professor, Department of Medicine, KMC/MGM Hospital, Warangal Dist., Telangana. ${ }^{2}$ Associate Professor, Department of Medicine, KMC/MGM Hospital, Warangal Dist., Telangana. ${ }^{3}$ Postgraduate, Department of Medicine, KMC/MGM Hospital, Warangal Dist., Telangana.

\begin{abstract}
Left ventricular function is the best individual predictor of mortality after acute myocardial infarction. After Acute Myocardial Infarction (AMI), a patient's prognosis is closely related to the extent of irreversibly damaged myocardium. The evaluation of infarct size after Acute Myocardial Infarction (AMI) is important for predicting the subsequent clinical course and to validate the effectiveness and clinical relevance of therapeutic interventions.
\end{abstract}

\section{REVIEW OF LITERATURE}

Coronary artery disease is the leading cause of death worldwide. The global burden of cardiovascular disease is expected to increase in the coming years, as falling mortality rates from coronary artery disease in the Western world are more than offset by the continuing epidemiological transition in developing countries away from nutritional deficiencies and infectious disease towards chronic and degenerative pathologies, cardiovascular disease being the most prominent.

\section{MATERIALS AND METHODS}

The study was carried out in a study group of consecutive 88 patients admitted in ICCU from March 2012 to August 2013 in the Department of Medicine of MAHATMA GANDHI MEMORIAL HOSPITAL, Warangal, satisfying the selection criteria (as per inclusion and exclusion criteria laid down).

\section{RESULTS}

The study was carried out in a study group of consecutive 88 patients admitted in ICCU from March 2012 to August 2013 in the Department of Medicine of MAHATMA GANDHI MEMORIAL HOSPITAL, Warangal, satisfying the selection criteria (as per inclusion and exclusion criteria laid down).

\section{CONCLUSION}

Serum troponin $\mathrm{T}$ concentration has a strong negative correlation with left ventricular ejection fraction after first acute myocardial infarction and hence can be used to assess the LVEF in patients with first myocardial infarction. A level of $>3.24 \mu \mathrm{g} / \mathrm{mL}$ provided a good indication for LVEF below 50\% with a sensitivity of $91.67 \%$ (CI 80 to 97.7 ) and specificity of $92.5 \%$ (CI 79.6 to 98.4) and thus can identify patients with higher risk.

\section{KEYWORDS}

Acute Myocardial Infarction.

HOW TO CITE THIS ARTICLE: Bhaskar P, Rao B, Naveen. Assessment of left ventricular function using 12-lead ECG and cardiac troponin-T in correlation with 2D-Echo following new-onset myocardial infarction. J. Evolution Med. Dent. Sci. 2016;5(64): 45574563, DOI: $10.14260 /$ jemds/2016/1040

\section{INTRODUCTION}

Left ventricular function is the best individual predictor of mortality after acute myocardial Infarction. After Acute Myocardial Infarction (AMI), the patient's prognosis is closely related to the extent of irreversibly damaged myocardium.(1,2) The evaluation of infarct size after Acute Myocardial Infarction (AMI) is important for predicting the subsequent clinical course and to validate the effectiveness and clinical relevance of therapeutic interventions.(3-5)

Financial or Other, Competing Interest: None.

Submission 16-07-2016, Peer Review 30-07-2016,

Acceptance 03-08-2016, Published 11-08-2016.

Corresponding Author:

Peddi Bhaskar,

Flat No. 303, Door No. 13-1-133,

Sai Towers, Matwada,

Warangal- Dist.,

Telangana State.

E-mail: bhaskar_peddi2003@yahoo.co.in

DOI: $10.14260 /$ jemds/2016/1040
Quantitative histologic estimates of infarct size are regarded as the gold standard, but the method has little clinical relevance. It is desirable to find a simple and reliable method with which to quantify infarct size.(6,7) Various methods such as electrocardiography, echocardiography, left ventriculography, radionuclide-based measurements and the release of cardiac biomarkers have been proposed.(8)

In clinical practice, the extent of injury to the myocardium after AMI is generally assessed by creatine kinase MB isoenzyme (CK-MB) release curves using serial serum sampling. ${ }^{(9,10)}$ Although quantitative calculations based on the area under the CK-MB vs. time curve are seldom made, many physicians use peak CK-MB to get a qualitative estimate of the size of the infarct.(11,12) The well-known limitations of CK-MB measurements, such as the short duration of increase after AMI the requirement for repetitive, frequent sampling for evaluation of peak concentrations, the sensitivity to reperfusion status and the lack of specificity for cardiac damage have stimulated the search for a more suitable biomarker for sizing infarcts.(13)

Cardiac troponin $\mathrm{T}$ (cTnT) is a cardiac-specific protein 
that is compartmented in the contractile apparatus of the myocardial cell. Its release process into the blood after myocardial injury is slow (cTnT is present in plasma for more than 120 hrs. after AMI), and it is only slightly affected by reperfusion of the infarct zone.(14) For these reasons, plasma cTnT has been used for estimation of infarct size.(15-18)

The cardiac Troponin $\mathrm{T}$ (cTnT) has been found to have excellent sensitivity and specificity and is superior to creatine kinase-MB (CK-MB) as indicator of myocardial necrosis. cTnT is uniquely located in the myocardium and its release closely relates to infarct size; therefore, inversely correlates with left ventricular ejection fraction (LVEF). We performed this study to find out the level of cTnT after AMI, and its correlation with LVEF.

The 12-lead Surface Electrocardiogram (ECG) is cheap, safe, quick, universally available and well-tolerated by patients. The Selvester QRS score can be applied to the 12lead ECG once the acute ST-segment deviation has resolved to estimate infarct size in both anterior and inferior ventricular locations.(19) From the QRS score, the LVEF can be estimated.(20) Both the QRS score and the subsequent LVEF calculation were however derived in non-reperfused infarcts. Several studies in reperfused infarcts have shown significant correlations between the QRS score, radionuclear perfusion defects and echocardiographic dyssynergy indices. The ECG QRS scoring included the complete 50-criteria, 32-point Selvester scoring system that has been previously reported and validated.

In this study, we applied the QRS score to patients surviving a first acute myocardial infarction. The purpose of the study was to compare the QRS score with left ventricular ejection fraction, relate the score to clinical and biochemical estimates of LVEF.

Coronary artery disease is the leading cause of death worldwide. The global burden of cardiovascular disease is expected to increase in the coming years as falling mortality rates from coronary artery disease in the Western world are more than offset by the continuing epidemiological transition in developing countries away from nutritional deficiencies and infectious disease towards chronic and degenerative pathologies, cardiovascular disease being the most prominent.

Acute MI is a serious and potentially lethal manifestation of coronary artery disease afflicting more than 7 million people worldwide each year. James Herrick established MI as a distinct clinical entity in 1912 and also installed the mainstay management strategy - which prevailed for the next 50 years - in stressing the importance of "absolute bed rest." Thanks to a remarkable scientific journey throughout the last 60 years - spanning epidemiology, basic science and clinical trials - such long-held beliefs are now considered obsolete; and a comprehensive, continually evolving evidence base has been generated from which contemporary preventive and therapeutic strategies have been developed.

Beginning in the late 1940s, prospective studies were designed to define lifestyle, environmental and other factors contributing to the incidence of MI. In the 1960s, designated coronary care units were established in many hospitals to monitor AMI patients and ensure prompt resuscitation in the event of life-threatening arrhythmias. A development called the "single most important advance in the treatment of acute MI." These significant progressions were then augmented by basic science studies elucidating many of the key underlying mechanisms of AMI, which paved the way for landmark clinical trials spanning from acute interventions such as reperfusion therapy to long-term pharmacological therapies that are now cornerstones in the management of AMI patients. The combined impact of these preventive and therapeutic measures has resulted in large reductions in mortality following AMI in the developed world.

In STEMI, the last innovation to provide clear-cut incremental benefit has been the introduction of PPCI. When delivered in a timely fashion, PPCI reduces early death, reinfarction and stroke compared to pharmacological reperfusion by fibrinolysis. Further therapeutic innovations and effective preventive strategies are still being pursued. Among these are efforts to protect the ischaemic myocardium against reperfusion injury. Reperfusion injury refers to tissue damage occurring as a consequence of blood supply being reestablished after a period of ischaemia. Mitigation of reperfusion injury has been demonstrated in animal models, but proved difficult to replicate in clinical studies. Several approaches have been tested, but none have yet demonstrated efficacy in pivotal clinical trials. The FIRE (Efficacy of FX06 in the Prevention of Myocardial Reperfusion Injury) trial from which database thesis is based, also failed to replicate experimental findings in a clinical setting. The concept of post-conditioning has emerged as one of the most promising strategies to confer cardio-protection in the setting of reperfusion. It is currently being evaluated in multiple randomised trials. So far results have been encouraging, but benefits may be restricted to patients with large infarctions.

Despite the significant improvements in prognosis, a gradient of risk still exists following AMI and risk stratification remains crucial to allocate resources efficiently, optimise patient outcomes and limit adverse events. Particularly, early identification of high-risk patients with extensive myocardial injury is important to ensure appropriate administration of pharmacotherapies and prophylactic interventions.

\section{MATERIALS AND METHODS}

The study was carried out in a study group of consecutive 88 patients admitted in ICCU from March 2012 to August 2013 in the Department of Medicine of MAHATMA GANDHI MEMORIAL HOSPITAL, Warangal, satisfying the selection criteria (as per inclusion and exclusion criteria laid down).

\section{Inclusion Criteria}

Patients who satisfy the WHO criteria for the diagnosis of acute MI are included.

- A history of ischaemic type of chest pain,

- Evolutionary changes on serially obtained ECG tracings, and

- A rise and fall in serum cardiac markers.

\section{Exclusion Criteria}

Patients Presenting with,

- Previous history of MI

- LVH, intraventricular conduction defects and complete heart blocks

- Valvular heart disease

- Cardiomyopathy

- Pericardial diseases 
- Congenital heart disease

- Previous cardiac surgeries, and

- Renal failure were excluded from the study.

No control subjects were taken, because cTnT is not detected in the peripheral circulation under normal circumstances.

Serum troponin- $T$ concentration was measured between 12-48 hours after the onset of chest pain.

Standard 12-lead electrocardiograms were recorded at a $25 \mathrm{~mm}$ paper speed for the patients who met the preceding entry criteria had a QRS score calculated from the discharge electrocardiogram on the basis of $\mathrm{Q}$ and $\mathrm{R}$ wave duration and $\mathrm{R}$ to $\mathrm{Q}$ and $\mathrm{R}$ to $\mathrm{S}$ amplitude ratios.

Echocardiograms were obtained using an echocardiographic machine, a $3.5 \mathrm{MHz}$ multiphase array probe in subjects lying in the left lateral decubitus position and supine position.

The echocardiographic techniques and calculations of different cardiac dimensions were performed according to the recommendations of the American Society of Echocardiography. The ejection fraction was obtained using an M Mode method from apical four chamber view. Measurements were made from three consecutive beats and the average of three beats was used for analysis. LVEF less than $50 \%$ was taken as systolic dysfunction.

The relation between LVEF and cTnT concentration; LVEF and QRS score was studied using Pearson's correlation coefficient and by systemic analysis of sensitivity and specificity.

Patients were initially categorised into two data sets, those with $\mathrm{EF}<50 \%$ and those with $\mathrm{EF}>50 \%$. Fisher's exact test or linear regression analysis were applied when appropriate. The most advantageous cut-off values to predict mortality were selected from visual inspection of the Receiver-Operator Characteristic curves (ROC).

\section{RESULTS}

The study was carried out in a study group of consecutive 88 patients admitted in ICCU from March 2012 to August 2013 in the Department of Medicine of MAHATMA GANDHI MEMORIAL HOSPITAL, Warangal, satisfying the selection criteria (as per inclusion and exclusion criteria laid down).

Mean age of the patients in present study was $52 \pm 12$. Most of the patients in our study were in age group 41-60 yrs. (82.5\%); $66.6 \%$ of patients were male and only $36.3 \%$ were female; $67 \%$ of patients had AWMI and $33 \%$ patients had IWMI, $60 \%$ patients had dyslipidaemia. It was found to be most common risk factor. Hypertension (57\%) and Diabetes (42\%) were other significant risk factors, mainly in male patients. Risk factors were much more common in AWMI as compared to IWMI patients (Table I).

\begin{tabular}{|c|c|}
\hline Variables & Values \\
\hline Age (yrs.) & $52 \pm 12$ \\
\hline Sex (M/F) & $56 / 32$ \\
\hline RWMA (Ant/Inf) & $59 / 29$ \\
\hline Thrombolysis (\%) & 82.9 \\
\hline Dyslipidaemia (\%) & 60 \\
\hline Hypertension (\%) & 57 \\
\hline Diabetes Mellitus (\%) & 42 \\
\hline Smoking (\%) & 28 \\
\hline Alcohol (\%) & 51 \\
\hline
\end{tabular}

\begin{tabular}{|c|c|}
\hline Cholesterol (mg/dL) & $147.7 \pm 66$ \\
\hline Serum Triglyceride (mg/dL) & $139.8 \pm 41$ \\
\hline Serum LDL (mg/dL) & $84.5 \pm 27.3$ \\
\hline Serum HDL (mg/dL) & $38.3 \pm 11.8$ \\
\hline Serum VLDL (mg/dL) & $32 \pm 13.2$ \\
\hline \multicolumn{2}{|c|}{ Table I: Clinical and Biochemical } \\
Characteristics of the Study Group \\
\hline
\end{tabular}

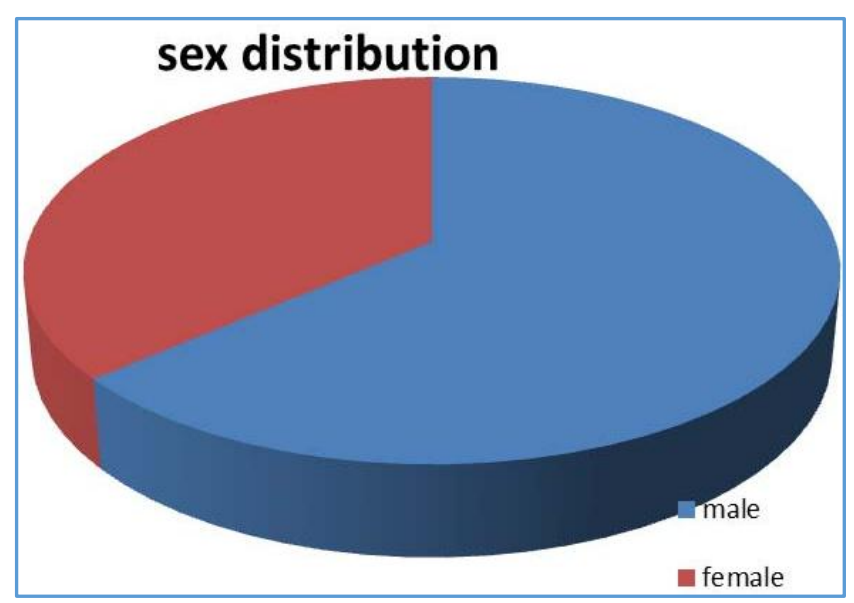

Fig. 1: Sex Distribution of My Study Population

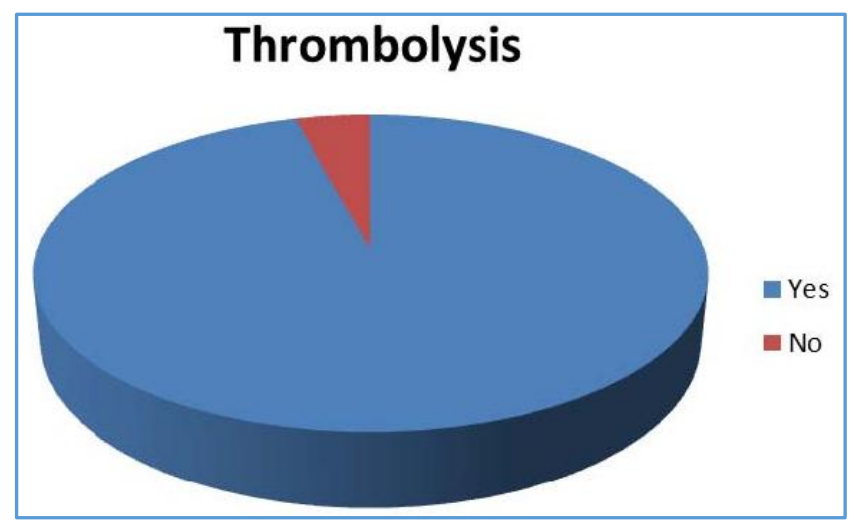

Fig. 2: Thrombolysed Cases

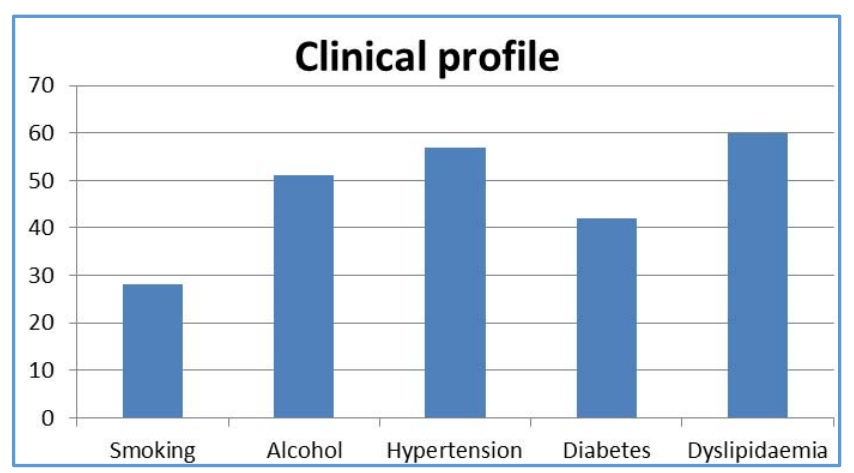

Fig. 3: Clinical Profile of My Study Population 


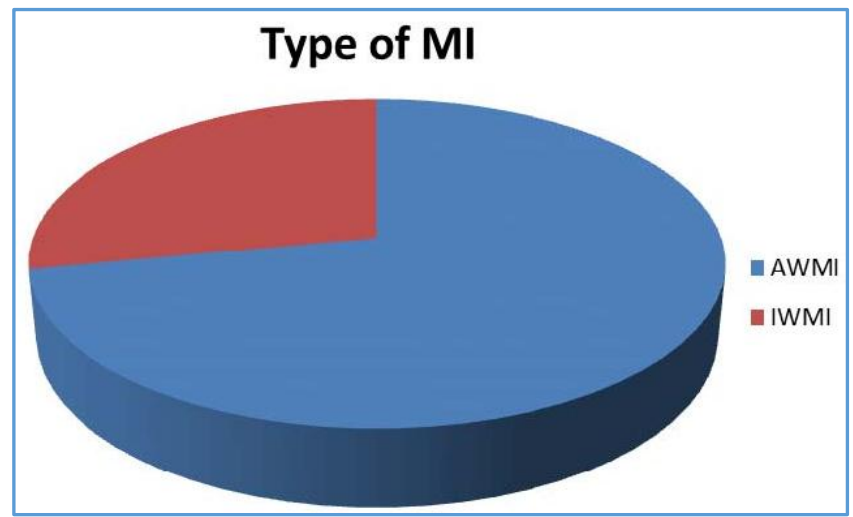

Fig. 4: Type of MI

When we assessed the relationship between cTnT and the indices of $\mathrm{LV}$ function obtained by Echocardiography performed at the time of ICCU discharge, cTnT was inversely correlated with LVEF ( $r=-0.4864 ; \mathrm{P}<0.0001$; Fig. 5).



Fig. 5

Table II shows the relation between cTnT and LVEF. There was a strong negative correlation between cTnT level and Echocardiographic LVEF. The Pearson's correlation coefficient between cTnT and LVEF was $r=-0.4864$ (Fig. 17). The cTnT value was high among patients with LVEF $<50 \%$, which was statistically significant ( $p<0.0001)$.

\begin{tabular}{|c|c|c|}
\hline Ejection Fraction & $\mathbf{N}$ & Trop. T $\boldsymbol{\mu g} / \mathbf{m L}$ (mean + SD) \\
\hline$<50 \%$ & 47 & $7.91+5.82$ \\
\hline$\geq 50 \%$ & 41 & $1.52+2.37$ \\
\hline \multicolumn{3}{|c|}{ Table II: cTnT Levels (Mean \pm SD) in Relation } \\
to Ejection Fraction \\
\hline
\end{tabular}

$(\mathrm{p}<0.0001)$

Analysis by ROC curve produced an area under the curve of 0.945 (95\% CI0.875 to 0.982$)$ at a cut-off left ventricular ejection fraction of $50 \%$ (Fig. 18). A troponin concentration of $>3.24 \mu \mathrm{g} / \mathrm{mL}$ predicted a left ventricular ejection fraction of $<50 \%$ with a sensitivity of $91.67 \%$ (CI 80 to 97.7 ) and specificity of $92.5 \%$ (CI 79.6 to 98.4 ).

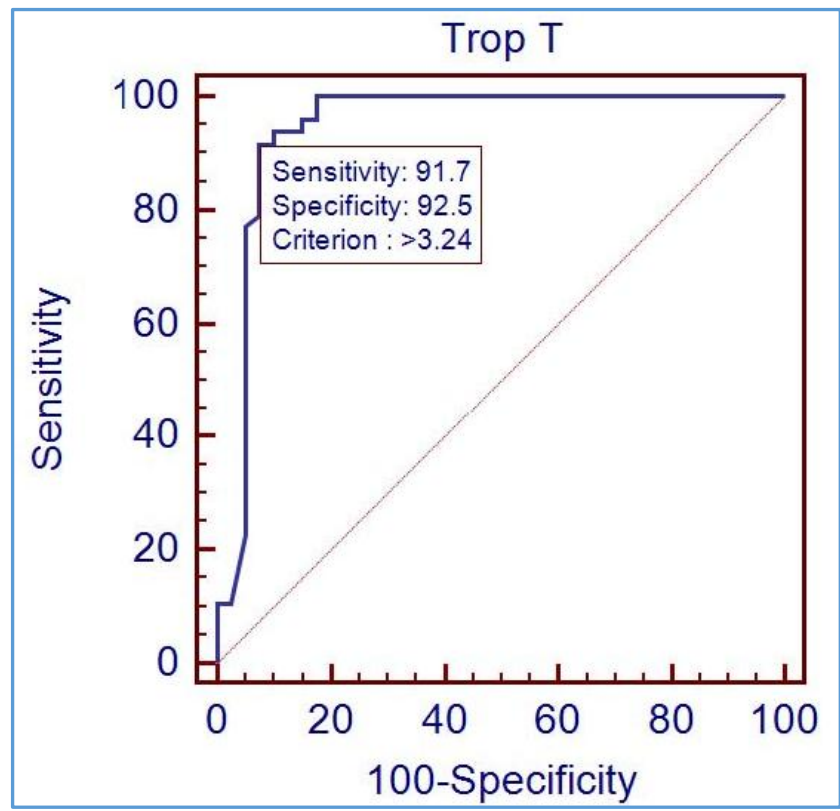

The result of re-analysis to examine the effect of reperfusion by excluding 15 patients who did not receive thrombolysis was no different from analysis of all patients.

Table IV shows the sensitivity and specificity of cTnT to predict LVEF. It was found that cTnT concentration $>3.24$ $\mu \mathrm{g} / \mathrm{mL}$ predicted LVEF of $<50 \%$ with a sensitivity of $91.67 \%$ and specificity of $92.5 \%$.

\begin{tabular}{|c|c|c|}
\hline Troponin T & EF $<\mathbf{5 0 \%}$ & EF $>\mathbf{5 0 \%}$ \\
\hline$\geq 3.24$ & $43(\mathrm{a})$ & $4(\mathrm{~b})$ \\
\hline$<3.24$ & $4(\mathrm{c})$ & $37(\mathrm{~d})$ \\
\hline \multicolumn{2}{|c|}{ Table III: cTnT Level and Ejection Fraction } \\
\hline
\end{tabular}

$\mathrm{a}=$ true positive, $\mathrm{c}=$ false negative, $\mathrm{b}=$ false positive, $\mathrm{d}=$ true negative

When we assessed the relationships between QRS score and the indices of LV function obtained by Echocardiography performed at the time of ICCU discharge, QRS Score was inversely correlated with LVEF ( $r=-0.5834 ; \mathrm{P}<0.0001$; Fig. 6)

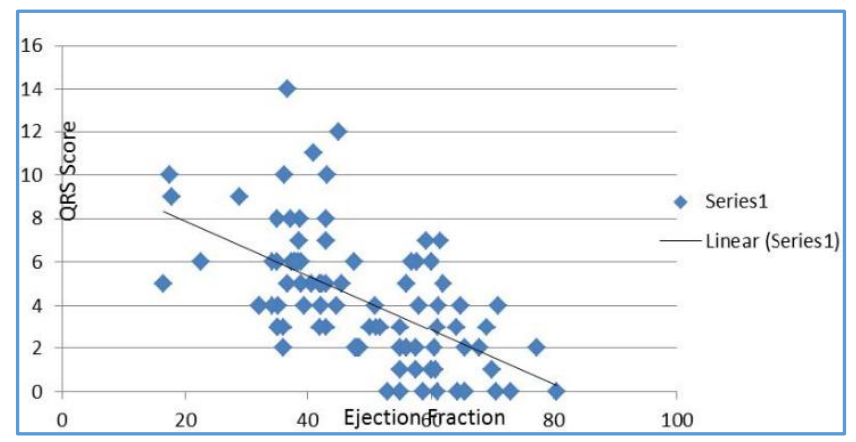

Fig. 6

AMI.

Table IV shows the relation between QRS Score and LVEF. There was a negative correlation between QRS score and LVEF. The Pearson's correlation coefficient between QRS Score and LVEF was $r=-0.5834$. The QRS Score was high among patients with LVEF $<50 \%$, which was statistically significant $(\mathrm{p}<0.0001)$. 


\begin{tabular}{|c|c|c|}
\hline Ejection Fraction & $\mathbf{N}$ & QRS Score (Mean + SD) \\
\hline$<50 \%$ & 47 & $5.8+2.7$ \\
\hline$\geq 50 \%$ & 41 & $2.4+2.1$ \\
\hline \multicolumn{2}{|c|}{ Table IV: QRS Score (Mean $\mathbf{S}$ SD) in } \\
Relation to Ejection Fraction \\
\hline
\end{tabular}

$(\mathrm{p}<0.0001)$

Analysis by ROC curve produced an area under the curve of 0.831 (95\% CI0.736 to 0.903 ) at a cut-off left ventricular ejection fraction of $50 \%$ (Fig. 20). The QRS Score of $>6$ predicted a left ventricular ejection fraction of $<50 \%$ with a sensitivity of $79.2 \%$ (CI 65 to 89.5 ) and specificity of $70.2 \%$ (CI 53.5 to 83.4).

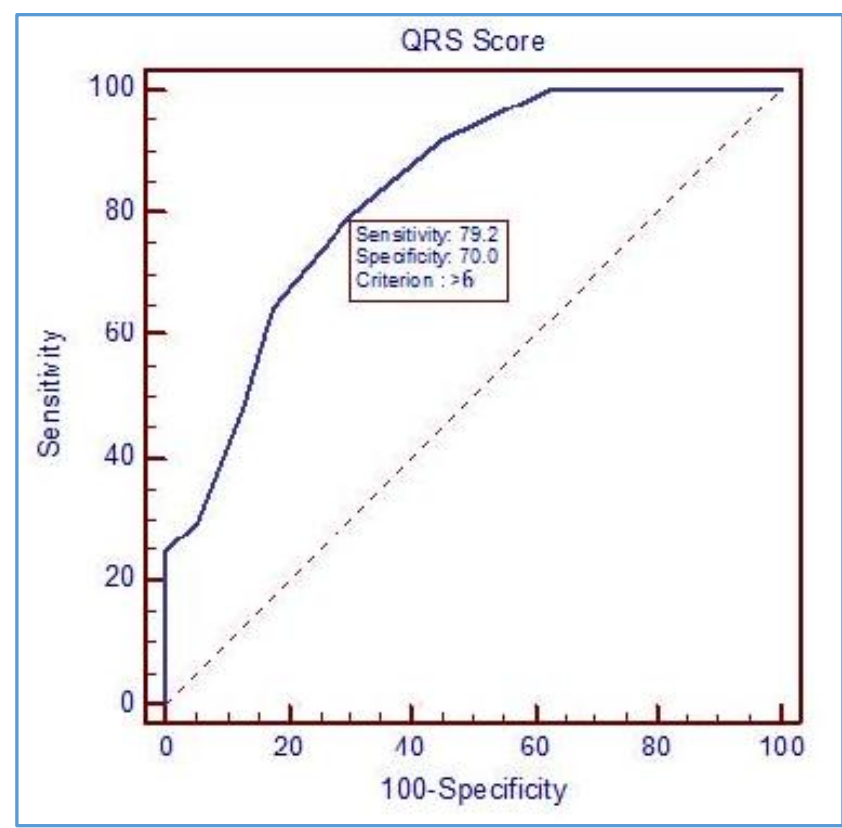

Table V shows the sensitivity and specificity of QRS Score to predict LVEF. It was found that QRS Score $>6$ predicted LVEF of $<50 \%$ with a sensitivity of $79.2 \%$ and specificity of $70.2 \%$.

\begin{tabular}{|c|c|c|}
\hline QRS Score & EF $<\mathbf{5 0 \%}$ & EF $>\mathbf{5 0 \%}$ \\
\hline$\geq 6$ & $43(\mathrm{a})$ & $19(\mathrm{~b})$ \\
\hline$<6$ & $4(\mathrm{c})$ & $22(\mathrm{~d})$ \\
\hline \multicolumn{2}{|c|}{ Table V: QRS Score and Ejection Fraction } \\
\hline
\end{tabular}

$\mathrm{a}=$ true positive, $\mathrm{c}=$ false negative, $\mathrm{b}=$ false positive, $\mathrm{d}=$ true negative

\section{QRS Score and Prediction of Ejection Fraction}

Of the 88 patients whose predicted left ventricular ejection fraction was compared with subsequent measured ejection fraction. Left ventricular ejection fraction was $47.1 \%$ $\pm 8.9 \%$ by ECG versus $48.6 \% \pm 13.8 \%$ by Echocardiography.

The correlation between predicted and measured ejection fraction is $r=0.5834[p<0.0001]$ (Fig. No. 7).

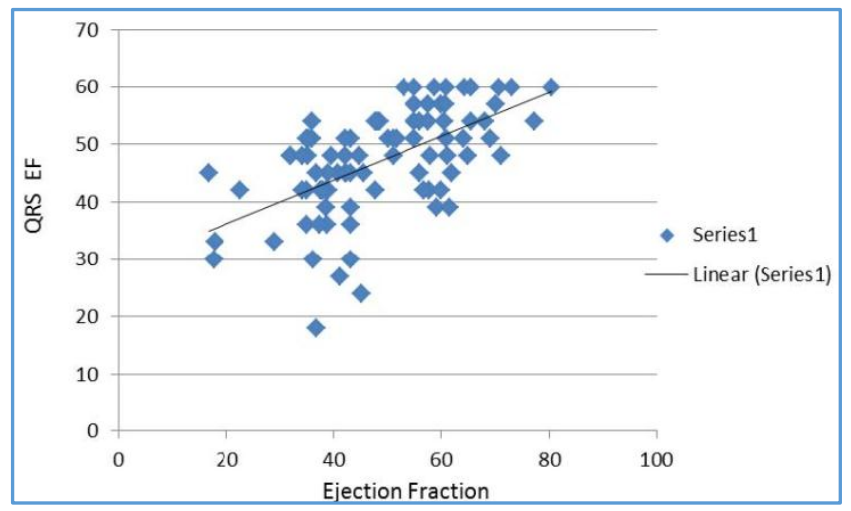

Fig. 7

\section{DISCUSSION}

Serum troponin $\mathrm{T}$ is accepted as a highly reliable biochemical marker for detecting myocardial damage and its use in the diagnosis of acute myocardial infarction is increasing.

Data show that serum troponin $\mathrm{T}$ is related to the amount of myocardial damage. But the relation between serum troponin $\mathrm{T}$ and impairment of left ventricular function after acute myocardial infarction has not been examined.

This study shows a strong negative correlation between serum troponin $\mathrm{T}$ concentration measured 12-48 hours postmyocardial infarction and echocardiographic left ventricular ejection fraction ( $\mathrm{r}=-0.4864 ; \mathrm{p}<0.0001$ ).

Analysis of the relation between troponin $\mathrm{T}$ and Echocardiographic left ventricular ejection fraction by ROC curve shows that a troponin $\mathrm{T}$ concentration of $>3.24 \mu \mathrm{g} / \mathrm{mL}$ is a sensitive (91.67\%) and specific (92.5\%) indicator of a left ventricular ejection fraction of $<50 \%$ after a first myocardial infarction.

The result of re-analysis to examine the effect of reperfusion by excluding 15 patients who did not receive thrombolysis was no different from analysis of all patients.

Troponin $\mathrm{T}$ has practical advantages over other markers in the assessment of left ventricular ejection fraction. After acute infarction troponin $\mathrm{T}$ has a peak value at 12 hours from the onset of pain, if successful reperfusion has occurred corresponding to washout of the cytoplasmic fraction. The plateau phase of troponin $\mathrm{T}$, however, lasts up to 48 hours and represents an integrated estimate of myocyte necrosis. The peak value will therefore be missed in samples taken $12-$ 48 hours after admission, but there is a large time window. This makes repeated sampling unnecessary and represents a cost and time effective method of diagnosis and quantification.

This is in contrast to creatine kinase MB or myoglobin for which multiple measurements are required and whose values are affected by thrombolysis.

In our study, cTnT levels closely correlated with Echocardiographic Ejection Fraction.

Our results corroborate those of earlier clinical studies in which cTnT release, not a single-point measurement was used to assess infarct size.

Furthermore, our findings in living patients are consistent with those obtained experimentally in dogs by Remppis et al(17) who found a good correlation ( $\mathrm{r}$ 0.69; P 0.003; $\mathrm{n} 16$ ) between cTnT concentrations 96 hrs. after the onset of ischaemia and the pathoanatomic infarct size as quantified by the 2, 3, 5-triphenyltetrazolium chloride method. 
In clinical practice, estimation of AMI size based on cTnT determination on a single plasma sample at CCU would facilitate the choice of appropriate care, leading to more efficient and economic use of healthcare facilities. This approach appears to be more useful than analysing cumulative cTnT release, as proposed previously because of the requirement of repetitive sampling and a possible incomplete recovery of cTnT.

LVEF is a very powerful prognostic indicator after AMI. A strong inverse relationship exists between LV function and patient outcome with rapidly increasing mortality rates at LVEFs $40 \%$.

Rao et al first showed a good correlation between cTnT concentrations measured 12-48 hrs. after admission and LVEF (r-0.72; P 0.001; n 50). In the study, a cTnT $2.8 \mathrm{~g} / \mathrm{L}$ predicted an LVEF $40 \%$ with a sensitivity of $100 \%$ and specificity of $93 \%$ (Area under the ROC curve, 0.98).

Kanna et al confirmed that serum cTnT on day 3 or 4 after AMI was significantly negatively correlated with LVEF assessed 1 month later (r-0.48; P 0.001; n 86).

Panteghini et al demonstrated a significant inverse relationship between LVEF derived from gated SPECT and plasma cTnT (r-0.56; P 0.001; n 65).

In this study, we got a significant correlation between QRS score and echocardiographic ejection fraction measured at the time of hospital discharge. There was a negative correlation between QRS Score and LVEF. The Pearson's correlation coefficient between QRS score and LVEF was $r=$ 0.5834. The QRS Score was high among patients with LVEF < $50 \%$, which was statistically significant $(\mathrm{p}<0.0001)$. The QRS Score of $>6$ predicted a left ventricular ejection fraction of $<$ $50 \%$ with a sensitivity of $79.2 \%$ (CI 65 to 89.5 ) and specificity of $70.2 \%$ (CI 53.5 to 83.4 ).

As expected, the QRS score is also related to estimates of left ventricular damage made by cardiac markers. Because QRS score correlates well with left five-year survival rate of $88 \%$; patients with a score of 10 or more had survival rates of 81 and $52 \%$ respectively at the same intervals.

The advantage of the QRS score is that it is calculated from a routine 12-lead electrocardiogram. It can be derived without risk in any patient who does not have left ventricular hypertrophy or conduction defects.

The QRS scoring system, which takes into account the duration and amplitude ratios of the QRS complex appears to be a better predictor of ejection fraction.

In this study, we used the QRS score in patients with a first infarction; it is still unclear whether it is as useful in patients who have had more than one infarction.

\section{CONCLUSION}

Serum troponin- $\mathrm{T}$ concentration has a strong negative correlation with left ventricular ejection fraction after first acute myocardial infarction, and hence can be used to assess the LVEF in patients with first myocardial infarction. A level of $>3.24 \mu \mathrm{g} / \mathrm{mL}$ provided a good indication for LVEF below $50 \%$ with a sensitivity of $91.67 \%$ (CI 80 to 97.7) and specificity of $92.5 \%$ (CI 79.6 to 98.4 ) and thus can identify patients with higher risk.

A significant negative correlation was observed between the QRS score obtained at the time of discharge and left ventricular ejection fraction $(\mathrm{r}=-0.5834)$. The QRS Score was high among patients with $\mathrm{LVEF}<50 \%$ that is in patients with a QRS score $\geq 6$. Left Ventricular Ejection Fraction was found to be $<50 \%$ with sensitivity of 79.2 and specificity of $70.2 \%$.

\section{REFERENCES}

1. Mair J, Friendrich L, Galzolari C. cTnI release correlates with myocardial infarct size. Eur J Clin Biochemistry 1996;335(18):1342-9.

2. Geltman EM, Ehanni AA, Campbell MK. The influence of location and extent of myocardial infarction on long term ventricular contractile dysfunction and mortality. Circulation 1976;60(4):805-14.

3. Sobel BE, Bresnahan GF, Shell WE, et al. Estimation of infarct size in man and its relation to prognosis. Circulation 1972;46(4):640-8.

4. Vassanelli C, Menegatti G, Nidasio GP, et al. Comparison of different pharmacological interventions on enzymatic parameters during acute myocardial infarction. Clin Biochem 1987;20(6):441-7.

5. Christenson RH, Vollmer RT, Ohman EM, et al. Relation of temporal creatine kinase-MB release and outcome after thrombolytic therapy for acute myocardial infarction. Am J Cardiol 2000;85(5):543-7.

6. Hørder M, Petersen PH, Thygesen K, et al. Plasma enzymes in myocardial infarction. An appraisal of quantitative, clinical and pathophysiological information. Scand J Clin Lab Invest 1981;41(1):41-7.

7. Holman BL, Goldhaber SZ, Kirsch CM, et al. Measurement of infarct size using single photon emission computed tomography and technetium $99 \mathrm{~m}$ pyrophosphate: a description of the method and comparison with patients prognosis. Am J Cardiol 1982;50(3):503-11.

8. Kaul S. Assessing the myocardium after attempted reperfusion. Should we bother? Circulation 1998;98(7):625-7.

9. Grande $\mathrm{P}$, Hansen BF, Christiansen C, et al. Estimation of acute myocardial infarct size in man by serum CK-MB measurements. Circulation 1982;65(4):756-64.

10. Hackel DB, Reimer KA, Ideker RE, et al. Comparison of enzymatic and anatomic estimates of myocardial infarct size in man. Circulation 1984;70(5):824-35.

11. Grande P, Christiansen C, Alstrup K. Comparison of ASAT, CK, CK-MB, and LD for the estimation of acute myocardial infarct size in man. Clin Chim Acta 1983;128(2-3):329-35.

12. Burlina A, Rizzotti P, Plebani M, et al. CPK and CPK-MB in the early diagnosis of acute myocardial infarction and prediction of infarcted area. Clin Biochem 1984;17(6):356-61.

13. Al-Saady NM, Camm AJ. New soluble markers for assessment of infarct size. In: Kaski JC, Holt DW, eds. Myocardial damage. Early detection by novel biochemical markers. Dordrecht, Netherlands: Kluwer Academic Publishers 1998:137-48.

14. Katus HA, Remppis A, Scheffold T, et al. Intracellular compartmentation of cardiac troponin $\mathrm{T}$ and its release kinetics in patients with reperfused and non-reperfused myocardial infarction. Am J Cardiol 1991;67(16):1360-7.

15. Voss EM, Sharkey SW, Gernert AE, et al. Human and canine cardiac troponin $\mathrm{T}$ and creatine kinase-MB distribution in normal and diseases myocardium. Arch Pathol Lab Med 1995;119:799-806. 
16. Ricchiuti V, Sharkey SW, Murakami MM, et al. Cardiac troponin I and $\mathrm{T}$ alterations in dog hearts with myocardial infarction: correlation with infarct size. Am J Clin Pathol 1998;110(2):241-7.

17. Remppis A, Ehlermann P, Giannitsis E, et al. Cardiac troponin $\mathrm{T}$ levels at 96 hours reflect myocardial infarct size: a pathoanatomical study. Cardiology 2000;93(4): 249-53.

18. Hammerer-Lercher A, Metzler B, Mair J, et al. Cardiac troponin $\mathrm{T}$ closely correlates with infarct size in a murine model of acute myocardial infarction. Eur Heart J 2001;22(Suppl):674.
19. Selvester RH, Wagner JO, Rubin HB. In: Snellen H, Hemker $\mathrm{H}$, Hugenholtz $\mathrm{P}$, et al, eds. Quantitation of myocardial infarct size and location by electrocardiogram and vectorcardiogram. Boerhave course in quantitation in cardiology. Leyden, Netherlands: Leyden University Press 1972:p. 31.

20. Palmeri ST, Harrison DG, Cobb FR, et al. A QRS scoring system for assessing left ventricular function after myocardial infarction. N Engl J Med 1982;306(1):4-9. 